CULTURA E DESENVOLVIMENTO 


\section{GPDs y desplazamientos poblacionales: algunas claves para su comprensión como procesos sociales complejos}

\section{Leopoldo J Bartolomé}

Universidad Nacional de Misiones Misiones Argentina

leobart@invs unam edu ar 


\section{Resumen}

La tematica planteada por el desplazamiento forzoso de poblaciones debido a la construccion de grandes represas y otras obras hidraulicas ha sido siempre polemica pero la controversia a ese respecto ha crecido en los ultumos anos cuando la entera cuestion dıl hidrodesarrollo ha recibido ataques desde el punto de vista ecologico social e inclusive economico Las posiciones extremas entre quients se oponen a las represas piden la suspension inmediata de tales proyectos y la desactivacion de las represas existentes subrayando los antecedentes negativos de los umpactos ecologicos $y$ sociales de esos emprendimientos A su vez los partidarios del hidrodesarrollo recalcan la imperiosa necesidad social de la disponıbilıdad de energia para el desarrollo y la inexustencia de alternativas vaables (menos ecologicamente danmas) para la gencracion de la misma Esta controversia se ha agudızado tanto que no parece dejar lugar para posiciones mas matızadas Este trabajo se centra en los aspectos conceptuales para el analisis de los componentes de desplazamiento

reasentamiento de los proyectos que los ocasionan y argumenta en favor de la utılizacion de una perspectiva mas amplıa y mas teoricamente impregnada Los procesos de desplazamiento y reasentamiento implican por lo general penodos temporales relativamente largos y como procesos su desarrollo es altamente susceptible a los eventuales cambios en las condiciones muciales (se refiere a conceptos de la teoria del caos) Por consiguente los sistemas que generan sc comport an como sistemas no lineares y contienen un grado implicito de impredictibilidad Por otro lado esos procesos se han desarrollado $y$ evolucionado en campos domunados por las relaciones de poder y son de naturaleza esencialmente politica La ignorancia de este hecho solo puede conducir a malos entendidos y a una comprension defectuosa d $\epsilon$ los reales impactos de un proyecto

Palabras claves represas relocalizaciones procesos impredictıbilıdad
Abstract

The issue of forced displacement of populations caused by the construction of large dams and other hydraulc projects has always been controversial but th has grown in recent years since the enttre subject of hydro development has been questioned from ecological soctal and even econornic points of new Extreme postitons within those opposing dams call for an immediate ban on hydro projects and the decommissioning of existing ones stressing the negative ecological and social impacts of previous endeavors on the other hand those favoring dams highight the pressing need for generating entrgy for development and the unavallabilty (at least at present time) of less socially and ecologically damaging alternatives This controversy has become so politccally entangled that there seems to extst little room for more moderate posttions This paper focuses on the conceptual framework for the analysis of the displacement/ resettlement component of these projects and argues for the netd to utzlize a broader and more theorettcally oriented perspective Displacement and resettlement processes generally involve significant time periods and as processes their development is highly susceptible to changes in the intial conditions (referring to concepts of Chaos theory) Consequently they behave like nonlinear systems and contain an intrinsic degree of unpredictability Furthermore they have evolved in a field dominated by power relations and are essentially political in nature Ignoring this fact can only lead to serious misunderstandings and faulty assessments of the real impacts of a project

Keywords dams relocalization process unpredictability 


\section{La controversia acerca de las represas}

$\mathrm{Z}^{\text {l desplazamiento forzoso y/o el reasentamiento de grandes nume- }}$ 1 ros de personas causados por la construcción de grandes represas hidroelectricas, sı bien puede ser considerado como un fenomeno típıco del siglo XX, esta lejos de constiturr un hecho del pasado y acumula un lamentable registro de consecuencias negatıvas, incluyendo la violacion de los derechos humanos básıcos Mundialmente, mullones de personas se han visto desplazadas de sus hogares tradıcıonales, sin que recibıeran compensaciones adecuadas, e inclusıve sıendo victımas de violencia fisıca Así, el Banco Mundial (1994) estıma que tan sólo en el período 1986-93, entre 90 y 100 millones de personas fueron desplazadas en asociación con proyectos de desarrollo y que aproxımadamente diez millones se ven involucradas en tal situacion anualmente Tan sólo en China, el numero de personas desplazadas por represas es estımado en más de 10 millones para el período 195089 (ADB, 1999) En la India post independiente, al menos 14 millones de personas han sido desplazadas por represas entre 1950 y 1990 (Fernandes y Paranjpye 1997) Y estas cifras, en si apabullantes, no incluyen otras categorias de afectados, como aquellos que si bien no se veron obligados a desplazarse, perdieron parcial o totalmente sus recursos de subsistencia Esta ultıma categorıa rara vez contabilızada es probablemente muy superior a la de las personas efectivamente desplazadas

En los últımos años esta controversıa se ha vuelto álgıda, habıendose desarrollado un amplıo movimiento "antı-represas', de escala mundial y creciente influencia que ha llegado a demorar e inclusive impedir algunos proyectos ${ }^{1}$ A pesar de que los aspectos polemicos de las represas no se limitan al componente de desplazamiento poblacional e incluyen su misma viabilidad economica, los efectos sobre la población son evidentemente los mas dramátıcos En realıdad, las 
que subyacen a esta controversia son dos concepciones polarmente opuestas acerca de qué significa "desarrollo", lo que torna difícl sino imposible concliar ambas posiciones

Desde un lado de la controversia los críticos de las represas sostienen que la concepción de desarrollo en términos de aumentos en infraestructura, tecnología y producto bruto interno es inherentemente erronea y debe ser reemplazada por concepciones alternativas y más centradas en el mejoramiento de la calıdad de vida

Este rechazo a lo que podriamos llamar modelo "tecnocrático" de desarrollo ası como a la vision de ' bien publico que implıca, incluye el rechazo a la concepción del desplazamiento poblacional como un corolario inevitable del desarrollo De esta manera, el 'desarrollo", que en esta perspectıva está intımamente asociado con la volación de los derechos humanos y el deterioro del bienestar de los desplazados, es concebido como inherentemente deletereo De allı el rechazo de plano al desplazamiento poblacional conjuntamente con la perspectiva politica y economicamente explotativa del desarrollo de la que forma parte (Declaracion de Manıbelı, 1994)

Desde el otro lado, se argumenta que los proyectos unfraestructurales de gran escala, tales como las represas, resultan umprescindıbles para el desarrollo económico nacional, en la medıda en que proveen de energía, urrigación y otros factores necesarios no sólo para el crecimiento economico sino para el mejoramiento de la calıdad de vida de los sectores postergados Por lo tanto, tales proyectos sirven al "bien publico" en el sentido más estricto Ahora, sı para concretar estos objetivos de bien publico se hace lamentablemente necesario el desplazamiento de personas, tal desplazamiento resulta un componente "legítımo" del proyecto A partır de este reconocimiento, el problema central es cómo minımizar ese desplazamiento y mitıgar sus posıbles impactos negatıvos Asimismo se admite la posibilidad de que si el proyecto es adecuadamente planificado e implementado la población afectada por el mismo pueda beneficiarse e inclusive mejorar su situación con respecto a la situación previa (ICOLD, 1999)

Esta polarizacion de la discusión y el análısıs en térmunos dificilmente concilables torna incomoda la situacion de quienes no concordamos

\section{ILHA}


en analizar la problematica dentro de tal marco conceptual Por mi parte, junto con otros cientıficos sociales dedicados al estudıo de los umpactos de los grandes proyectos de desarrollo, considero que los desplazamientos poblacionales no son nı deben ser consecuencias inevitables del desarrollo y que, de resultar necesarı, el reasentamiento de los desplazados no tiene por que constituurse en una experiencia pauperızante ${ }^{2}$

Tan solo para definur un poco mas mi posición a este respecto no creo que sea productivo enmarcar estos temas en terminos de ser (ideológicamente) pro o contra el desarrollo economico o las grandes represas (Bartolomé 1992) En cambı, creo furmemente que el desarrollo debe posibilitar a sus eventuales afectados el acceso a un auto sostemible mejoramiento de sus a) curcunstancias mateuales, b) opciones para el reasentamento y c) control sobre las circunstancias de su vida cotıdiana Indudablemente, estos beneficios pueden ser logrados a traves de una vanledad de medios y no solo a traves de intervenciones técnicas de gran escala pero las mismas deben estar solidamente ancladas en el conocimiento y el respeto a las estructuras sociales y las practicas culturales de la población afectada Si bien los desplazamientos poblacionales forzosos son procesos inherentemente problemátıcos, la experıencia mundial enseña que cuando el reasentamiento no es suficientemente planificado e implementado en forma sensible y responsable, causa mucho sufrimiento a millones de personas A pesar de ello y bajo determınadas circunstancias, el reasentamiento, cuando planificado e implementado como una intervención orientada al desarrollo, puede converturse en una oportunidad excepcional para el mejoramiento de las condiciones de vida de poblaciones marginadas

Esto no significa que el logro de resultados positivos en un proceso de reasentamiento dependa fundamentalmente de una planıfıcacıón e implementacion eficientes Nunca debe olvidarse la presencia de elementos relacionados con el "poder" que son consustanciales con el proceso, con algunos actores poseyendo o de alguna manera controlando los medıos legales, admunistratıvos y de fuerza, que pueden obligar a otros a obedecer su voluntad y resignar los intereses propıos De allí que resulte crítıco para el logro de resultados positıvos en un proceso de

\section{ILHA}


reasentamiento el hacer que el "poder" sea accesible para los afectados, especialmente para los economica y socialmente marginados Y central para este objetivo es la manera en que se "negocie" y conduzca el proceso de reasentamiento, para lo cual se requieren no sólo prevssiones para el mejoramiento de las condiciones materiales de vida de la población afectada, sıno también el establecımuento de mecanısmos transparentes y efectivos para la defensa de sus derechos civles

La conclusión de que el reasentamiento debe ser concebido e implementado como una "intervencion para el desarrollo" -en el sentido antes definido es el resultado de muchos años de investigaciones y análısıs de experıencıas de grandes represas, conducıdas por muchos de los cientificos sociales mas destacados, como Thayer Scudder (1996, 1999 y otros trabajos) y Michael M Cernea (1999 y otros trabajos) ${ }^{3}$ Sin embargo, resulta necesario que traslademos (al menos temporariamente) la discusion fuera del marco de la ideológıcamente teñida controversıa que rodea actualmente el tema de las grandes represas, y que miremos el problema desde una perspectıva más amplıa Ello es necesano para aclarar ciertos temas que obstaculızan nuestra concepcion de la verdadera naturaleza de los procesos que nos ocupan, y distorsionan la evaluacion de los impactos sociales El primero de los puntos que debemos aclarar es a qué nos referımos al hablar de desplazamientos forzosos de población

\section{La naturaleza de los desplazamientos forzosos}

El desplazamiento forzoso de poblaciones ha sido una constante en la historia humana Ya sea por causas naturales (inundaciones, terremotos, sequías, etc ) o sociales y demográficas (guerras, sobrepoblaciones conflictos sociales, pobreza, etc), la gente se ha visto obligada a abandonar sus hogares tradıcıonales, generando así algunos de los eventos más importantes en la historia de la humanıdad sobre la tierra Desde esta perspectiva, los desplazamentos poblacionales resultantes de las llamadas "decisiones de desarrollo" (por ejemplo, la construcción de grandes represas) se ubican entre aquellos que reconocen causas sociales Sin embargo y a pesar de esta semejanza, los desplazamientos de este ultımo tipo se insertan claramente en campos polítıcos que involucran la operación de factores tales como el "poder 
social" y su distribucion, ası como otros atınentes al diverso grado de "agencia"4 disfrutado por los actores partıcipantes Este hecho convo ca a su vez conceptos tales como "legitumidad", "derechos colectivos versus indıviduales" "interes publıco", etc , que nos derıvan al campo de los valores y de la diversidad cultural

Poniendolo de otra manera no se puede negociar con una inundación o un terremoto, pero sí es posıble hacerlo -al menos potencialmente- con un gobıerno Por más pequeña que sea esa posıbılıdad de negociacion, su mera exıstencia potencial convierte a la decisıón de construur una represa (o cualquier proyecto de infraestructura) en una decısıon polítıca y dispara un proceso polítıco Este proceso posee la capacidad de determinar no solo el destıno de un proyecto, sino tambien la naturaleza de los impactos que han de ser sufridos por todos los participantes, voluntarios o involuntarios, del mismo

Y esta constatación nos conduce al punto central de mı argumento un proyecto de desarrollo, por ejemplo la construcción de una represa, no es que tenga implicaciones politicas sino que es en si mismo un proceso político Ignorar esto sıgnifica caer en una incomprensıón fatal acerca de la misma naturaleza del fenómeno que nos ocupa $\mathrm{La}$ tecnologia (y las soluciones tecnicas) existe en sociedad y tan pronto un factor tecnico afecta diferencialmente a una multıplicidad de actores sociales con intereses igualmente diversos, se convierte en un componente de un "campo' politico Consiguientemente, el desplazamiento y/o reasentamiento de poblaciones es también un producto de ese proceso politico y debe ser tratado desde esa perspectiva

\section{Adaptabilidad humana y las consecuencias del desplazamiento/ reasentamiento forzoso}

Afortunadamente para nosotros como especie, los seres humanos poseemos una notable capacidad de adaptacion y somos capaces de sobrevivir y aún prosperar bajo condiciones extremadamente desventajosas Estas capacıdades adaptatıvas son valıdas tanto frente al medıo ambiente natural como social, con el resultado de que muy pocos de los "escenarıos" naturales y sociales derivados del desplazamiento o del reasentamiento son totalmente negativos para la poblacion afectada, no al menos para la totalıdad de la misma 
Con el transcurso del tiempo, siempre existe gente capaz de extraer benefıcıos aún de las condiciones más desfavorables, aunque sea a expensas de otros afectados Este hecho dificulta la formulacion de evaluaciones globales de los resultados de un proceso ¿Bajo cuales parámetros podemos definur el éxito o el fracaso de un proyecto y en que "corte" temporal? Un mismo proyecto, por ejemplo, el reasentamiento de Mazatecos y otros campesinos indıgenas a raz de la construcción de la represa Cerro de Oro, en Méxıco, fue definıdo como "etnocıdı" por algunos investıgadores (Barabas y Bartolomé, 1973) y como un "exıto" parcial por otros reconocıdos expertos (Partrıdge y Brown, 1983 y 1984) Asımısmo, cl célebre caso de la población Nubia desplazada por la represa de Assuan, en Egıpto, pudo justıficadamente ser calıficado como un fracaso" hasta hace relatıvamente poco tiempo ( $\mathrm{y}$, de hecho, lo fue), mientras que en la actualıdad muchos lo consideran como "exitoso" desde varios puntos de vista

El hecho es que cualquier cambio en la estructura de oportunıdades -en su acepción económica- que caracterıza una situación dada, produce consecuencias que afectan diferencialmente las estrategias adaptativas de la poblacion involucrada En otras palabras, no hay cambios en las estructuras de oportunidades que no resulten en consecuencias, positivas o negativas para el grupo involucrado, pero la calıficacion de esos impactos puede resultar ampliamente divergente de acuerdo a la perspectiva de los evaluadores Ası, para aquellos que priorıcen la "integrıdad cultural" o la "homogeneıdad economıca" del grupo, un incremento de la diferenciación economica y en la orientacıon hacıa el mercado global, será considerado un indicador de consecuencias negatıvas mientras que para otros pueden ser interpretados como indicadores de un mejoramiento de la productividad economica $\mathrm{y}$, consiguientemente, de un "exito" en el proceso de reasentamento Estas consıderaciones Ilustran las dificultades exıstentes para alcanzar el objetıvo de evaluaciones verdaderamente "objetıvas' a partır de las cuales definır "buenas practıcas" (como se propone la Comision Mundıal de Represas) sın una discusıón y planıficacıon previa del significado del exito o del fracaso en este contexto 


\section{Actores sociales, impactos y el factor temporal}

Otra "trampa" muy común que conduce a otra conceptualızacion errónea de los desplazamientos poblacionales asociados con proyectos de desarrollo, radica en modelar los procesos como si los mismos unvolucrasen tan solo dos bloques solıdos y homogéneos los constructores (gobiernos, planificadores, empresas, etc ) y la poblacion afectada (los desplazados los reasentados, los pobres y sin poder, etc), ası como olvidar que los procesos se desarrollan en el tiempo y que a menudo se extienden sobre largos períodos ${ }^{5} \mathrm{Ni}$ la construccion de una gran represa nı la manifestacion de sus consecuencias sociales y naturales constituyen fenomenos instantaneos", sino eventos que ocurren en marcos temporales que son significativos en terminos de procesos historicos Esta dimension temporal implica que la conflguracion de factores que puede caracterizar a un proceso en su fase inicial, se encuentra abierta a alteraciones que pueden originarse no solo en su unterıor sino también en el exterıor, es decrr, en el contexto socioeconómico nacional o internacional, etc Para decirlo en pocas palabras, los procesos de desplazamiento y reasentamiento poblacional asociados con proyectos de desarrollo son en sı procesos histórıcos y el conocumiento de sus condiciones uniciales no garantıza el conocimiento acerca de su futuro

A pesar de que la naturaleza y la intensidad de los impactos de los grandes proyectos de desarrollo pueden ser muy diversas y muchas de ellas dependen de condiciones locales especificas, la experiencia mundial sugiere que las principales areas de impacto son

a) Las estrategıas de supervivencıa de la población afectada, un concepto que abarca muchas cosas, entre ellas, cosas tales como el mapa mental de recursos aptos para ser explotados, el conocimiento tradicional acerca de como explotar esos recursos y las redes sociales construıdas a ese efecto Esas estrategıas son conformadas a través de los procesos ajustativos y adaptatıvos implementados por una poblaclon dada en el proceso de definir su "nicho adaptatıvo" en termunos de la antropologia ecologica

b) Los mecanısmos para la apropiación sımbolıca del medıo ambiente natural y social significados asıgnados a objetos y paisajes, 
tales como montañas, grutas, ríos, etc , y a los espacios especiales resultantes de la accion social como, por ejemplo, cementerıos, lugares sagrados y sitıos de culto de diferente tipo, etc

c) El sistema de status y relaciones sociales que definen tanto la interacción efectıva entre un grupo y otros, ası como su auto percepcıon Este sistema uncluye no solo la distribución tradıcıonal de "poder social ' entre grupos e individuos, suno también las cuotas "percıbıdas" de "agencia' que se atribuyen

A pesar de que estas tres áreas de impacto estan lejos de agotar el universo de las consecuencias posibles del desplazamiento y el reasentamiento, cubren la mayoria de las que pueden ser generalizadas

Retornando a nuestra informacion acerca de la multiplicidad de actores sociales involucrados en los procesos de desplazamiento/ reasentamiento, es importante "desconstruir" la categoría "población afectada" El sumple hecho de ser afectado por un proyecto no define a un grupo en un sentıdo sociológıco, es decır, no implica la existencia de atributos sociales comunes ni de objetıvos comunes (de hecho, dichos objetıvos pueden ser totalmente contradıctorıos) A pesar de que este hecho pueda parecer obvio, la verdad es que muchos planuficadores asumen la existencia real de esa comunidad de intereses y esperan actitudes y respuestas congruentes con esa supuesta homogeneidad

Frecuentemente, las que aparecen como diferencias insıgnuficantes a los ojos de los planificadores, son percibidas como cruciales por los afectados, determinando actıtudes y estrategias muy diferentes Una anécdota ocurrida en el contexto del Proyecto Itaparica (Brasil), surve para ilustrar este punto ${ }^{6}$ Las diferencias sociales y economicas existentes entre los pequeños terratenientes que explotaban pequeñas huertas de irrigacion en los bancos nbereños del Río San Francisco y sus empleados (ambos de aspectos y condiciones de vida igualmente miserables) fueron consıderadas como muy menores y totalmente despreciables desde el punto de vista del diseño de soluciones para el reasentamiento Sin embargo, esas diferencias eran basicas para la compleja estructura polítıca local, basada en relaciones de patron - cliente y determunaron posicıonamientos ampliamente divergentes de cada grupo en relación al plan de reasentamıentos que se les estaba proponıendo Lo que para los 
trabajadores sin tierra significaba la posıbılıdad de acceder a la condıción de propıetarıos ( $\sin$ que importase la calıdad de la tierra recibıda), para los pequeños propıetarıos implıcaba la destruccion parcial sino total de sus estrategias de supervivencia y una erosion terminal de las bases de poder politico, por menguado que este fuere

Una lección a ser aprendıda a partur de éste y de numerosos otros casos es que ignorar las diferencias exıstentes entre la población afectada ( $y$, consecuentemente, en la constelacion de intereses percibidos que están activos en una situacion dada) en beneficıo de la rapidez, es probable que genere mayores problemas que aquellos considerados por las "soluciones" propuestas Los umpactos que afectan el área que antenormente designamos como c) pueden ser tanto o mas deletereos que aquellos que inciden sobre a) o b) Más aún, esta heterogeneidad de intereses se da asimismo entre los sectores supraordınados involucrados en un proyecto de gran escala, es decur, el gobierno, los sectores admunstrativos, empresariales, etc Por lo tanto, uno de los aspectos claves para el diseño de un plan de reasentamento adecuado precısa de los grupos e intereses involucrados y de la implementación de un proceso amplıo y partıcipatıvo de "negociacion" de las soluciones a ser aplicadas

\section{Conclusiones el desplazamiento/reasentamiento como proceso social}

Todas las consideraciones precedentes concurren a enfatızar el hecho de que tanto los desplazamientos no planıficados como los planificados (reasentamiento) deben ser considerados y analızados como procesos sociales y no como fenómenos aislados y singulares Todo proyecto particular sigue una trayectoria en el tiempo (to, tl, t2 tn) -que frecuentemente se cuenta en años y no pocas veces en decadas- a lo largo de la cual el sistema (compuesto por actores sociales, ielaciones, intereses objetivos explicitos e implícitos, recursos, etc ) puede adoptar, y de hecho adopta, diferentes configuraciones Los proyectos de desarrollo de gran escala son precisamente eso de gran escala, $y$ pueden faculmente atraer a su campo factores que no estaban presentes en sus estadios inıciales Esa elastıcidad de sus fronteras como proyecto implica que las relaciones entre los agentes partıcıpantes y aún el cuiso entero del proyecto pueden modıficarse drásticamente entre 
t0 y th Tal como afirmamos anteriormente, no solo son sociales por su naturaleza sino historicos por su ocurrencia

Ası, por ejemplo, el proyecto Akosombo poseyo una relevancia polítıca en la Ghana gobernada por N'krumah, que se desvaneció cuando cambıo la estructura polítıca de esa nacion De la misma manera, la insercion de la población Nubia en el cuerpo politico de Egıpto adquirió una forma e importancia muy diferente en el Egıpto post Nasser en comparación con la de los primeros estados de las relocalızaciones de Assuan, un cambio polıtıco que transformó drastıcamente el destuno de la poblacion ı easentada De manera simılar, procedımıentos que resultaban impensables bajo un regimen militar autontarıo pueden volverse obligatorıos bajo gobıernos democratıcos, como ilustra la historıa del proyecto Salto Grande (Argentına - Uruguay) (Catullo, 1996)

Escala, temporalidad y procesos de toma de decisiones politicamente anclados, nos llevan a subrayar otro aspecto de las esquemas de reasentamiento inducidos por el desarrollo el rol central desempeñado por la ideología y el poder La ideologıa determina el contenıdo de la nocion de desarrollo" y de las acciones consideradas instrumentales a esos fines Desde esta perspectıva más amplia, la construcción de grandes represas y otros mega proyectos de desarrollo se encuadran claramente dentro de lo que se ha dado en llamar cosmovisión "modernista", y se basa en nociones y motivaciones que, por lo general, resultan incomprensibles para la poblacion local directamente afectada Tales ıdeas y valores no sólo no son compartıdas sino que frecuentemente resultan totalmente inuntelıgıbles para la poblacion afectada, pertene ciente por lo general a los sectores menos privilegiados y muchas veces marginados de la sociedad global Este factor torna muy dificultoso el establecumiento de un dialogo a través de una distancia social tan grande y lo abre excesivamente a malentendıdos y dısonancias semıtıcas que convierten muchas veces a las negociaciones de partes en una comedia de enredos cCual puede ser el sıgnificado de conceptos tan abstractos como el de interes publico para un campesino minifundısta o un habitante tribal obligado a trasladarse de sus tierras tradicionales, cuando muchas veces ni siquiera es consciente de pertenecer a una sociedad más abarcadora y compleja? Esto me recuerda a la escena de 
la película 'Los caballeros de la mesa redonda", del grupo britanico Monty Python, en la que un exasperado Rey Arturo trata de que un campesino le informe quen es "el Señor de esas tıerras"

Por una parte, los actores involucrados en procesos de la clase que aqui nos ocupa, tienden a estar incluidos en sistemas politicos en los que la distribucion del "poder social" es muy desigual Este factor determina, a su vez, capacidades de "agencia" igualmente diferentes Estas desıgualdades se dan no solo entre "orıginadores" y afectados" sino tambien entre los mismos afectados directos Cuando prevalecen estas grandes diferencias en capacidad de agencia, los procesos de negociación, aunque se pretendan partıcıpatıvos, tıenden a convertırse en rituales sin sentıdo o a menudo en rituales cuyo verdadero objetivo es el de "complacer" a las agencias financiadoras internacionales que promueven la utılızación de metodologías partıcıpatıvas El bien conocıdo caso de la Fundación Pehuen, propuesto orıgınalmente como un mecanismo innovador en el contexto del reasentamiento de poblacion Pehuenche en el Proyecto Pangue (Chile), ilustra las limitaciones de las acciones "bienintencionadas" pero basadas en el supuesto de la existencla de intereses comunes entre "ovejas" $\mathrm{Y}$ "lobos' El objetıvo ultımo de los lobos es el de maxumuzar la ingestion de ovejas, mientras que el de las ovejas es mantenerse lo más lejos posible de los lobos ¿Cual es el punto medio entre esos intereses compuestos? $S_{1}$ es que existe uno, no es probable que se lo alcance sentando a ovejas y lobos juntos

La principal razon de por que es posible proponer tan pocos "universales" en los esquemas de reasentamiento radica en que los mismos son extremadamente dependientes del contexto y se desarrollan dentro de campos de fronteras fluctuantes De all también la difıcultad para desarrollar patrones evaluatıvos de valıdez unıversal y criterios concensuados para definur exito o fracaso, buenas practicas, etc Resulta crítico, por lo tanto, construir criterios claros que especifıquen resultados esperados, sólıdamente anclados en la naturaleza de estos procesos y lo menos ideológicos posibles A tal proposito, es necesarı exorcizar ciertos "idolos' -parafraseando a Francis Bacon (Gould, 2000)- que interfieren en nuestra comprension de los procesos de desplazamiento y reasentamiento 
Ejemplo de tales "ídolos" son

a) La suposición de que los impactos negatıvos pueden siempre ser compensados y/o mitigados

Toda intervencion externa en un sistema de estrategias de supervvencia modifica la estructura de oportunidades (recursos, restricciones, conocimıentos prácticos, etc ) y dado que esas estrategıas son producidas por famulias y/o individuos, resulta practicamente imposible replicar la situación previa Asımismo, es igualmente cierto que todo cambio en la estructura de oportunudades, sea debido a causas fisicas o sociales, resulta en un uncremento en la diferenciación social (Bartolomé y Baranger, 1966) Aún cuando las acciones de intervención tengan entre sus objetıvos mejorar la situación económica y social colectiva, los beneficios eventuales rara vez se distribuyen en forma pareja Frecuentemente ocurre que quenes mejoran son los que estaban en pruncipıo en mejores condiciones, mientras que quienes estaban mal, termman aun peor Desde este punto de vista, conviene que las acciones de la agencia responsable de la implementacion se focalicen en fortalecer la situación de los grupos de nesgo dentro de la poblacion afectada, tales como mujeres, nunos, ancianos, munonas etnicas relegadas, etc Es decir, aquellos que presuntıvamente habran de tener mayores problemas para reconstruir sus estrategıas de supervivencia frente al cambıo en la estructura de oportunıdades

b) La utılızacion de dicotomías sumplistas tales como "victumanos/ victımas", así como la "angelizacion" de los pobres y margınados

Por "angelızacion" me refiero a la ıdealızación extrema de un grupo social, denegandole inclusive atributos humanos como mentır, sentir envidia, aprovecharse de otros, etc Aunque pueda parecer ser lo contrarıo, esta actıtud representa en realıdad una forma de subestımación y de negar atributos específicamente humanos (aunque sean negatıvos) a grupos socialmente subordınados Esta actıtud puede conducir a errores muy peligrosos al tratar de entender y/o presidir acciones de la poblacion afectada Los "defectos' $\mathrm{Y}$ "Vicios" parecen estar distribuidos en forma pareja a lo largo de la escala social y los planificadores deben ser concientes de este hecho

c) Enmarcar el proceso de reasentamiento en terminos de una relación entre dos grupos supuestamente homogeneos y asumur que todos los afectados comparten intereses comunes 
Me remito a lo ya dicho a lo largo de esta ponencia

d) Asumir una naturaleza "tecnica" para el conjunto de acciones a ser consideradas en un esquema de reasentamiento y suponerlas undiferentes a la manıpulación polítıca y a la desıgual dıstrıbución e instrumentacion del poder social

Este es quizás uno de los mas persıstentes idolos" debido a que se conjuga con el sesgo profesıonal de la mayoria de los planuficadores, quienes se resisten a admitir que su trabajo no se encuentra basado en verdades absolutas y valores universales Las organuzaciones $\mathrm{fl}_{\mathrm{l}}$ nancieras internacionales a menudo refuerzan esta actitud al estar formalmente inhibidas para intervenir en asuntos "politicos" de un paus De allı su insıstencia en caracterızar como " técnicas" (quenendo significar no politicas) a las medidas que propugnan A pesar de que tal actitud puede ser comprendida y aun justificada desde el punto de vista de las reglas que gobiernan las relaciones internacionales, resultan uncongruentes y engañosas para el análısıs critıco (es decur, cıentıfico) de procesos sociales La utılızación de anteojeras puede ser funcional para conducir caballos unidos a un carro, pero nunca para la comprension de la conducta humana Por lo tanto el conocimiento preciso de la distribucion del poder y de los varıos grados de "agencia" unvolucrados en una situacion dada constıtuye no sólo un prerrequisito para estudıos de factıbılıdad y para la implementación de sıstemas de monitoreo efectivos, sino la verdadera clave para la comprensión de la naturaleza de los procesos que se encuentran bajo analisis

Funalmente quiero señalar la felız councidencia entre la postura que he expuesto en estas págınas y la de mı colega sudafricano Chrıs de Wet, quien en una reciente ponencia (2000) ha demostrado convincentemente la falsedad de un "ídolo que sı bien no ha sido expresamente mencionado en este trabajo, se encuentra implicito en el mismo la nocion de que todos pueden beneficiarse igualmente de los proyectos de desarrollo Por consiguiente, el desafío para quienes nos ocupamos de esta tematica es el de encontrar mecanismos de intervencion capaces y eficientes para la canalizacion de los potenciales "beneficıos" hacia los sectores menos privilegiados de las poblaciones afectadas

Posadas agosto 15 de 2008 


\section{Notas}

1 A raiz de la fuerza ganada por este movimiento y a causa de las criticas recibidas por la politica implementada por el Banco Mundial en 1997 se constituyo la Comision Mundial de Represas (World Comision on Dams) conformada por representantes del Banco Mundial organizaciones no gubernamentales afectados por las represas empresas constructoras $y$ academicos con la mision de realizar una evaluacion objetiva de los efectos de las grandes represas El unforme final es esperado para fines de este año y promete ser bastante polemico

2 Distingo aqui entre desplazamiento la eviccion de personas sin compensacion adecuada o intento de planificar el proceso y reasentamiento donde existe por lo menos la intencion de planıficar el proceso y mitigar sus consecuencias negativas

3 Ambos recientes recibientes de los Premıos de Malınowski para Antropologia Aplicada

4 Pol agencia se entıende la real capacidad de un agente social para influenciar sus circunstancias naturales y sociales mas alla de su asıgnacion formal de poder (Giddens 1984)

5 Por ejemplo el componente de reasentamiento del Proyecto Yacyreta (Argentina

Paraguay) se ha extendıdo ya por mas de veınte años y no da señales de finalızar pronto

6 Experiencia personal

\section{Biblıografía}

ASIAN DEVELOPMENT BANK China resettlement polictes and practices review and recommendations Regional Technical Assistance Project Manila Asıan Development Bank, 1999 Draft

BARABAS Alıcia BARTOLOME, Miguel A Hydraulic development and etnocide the Mazatec and Chinantec People of Oaxaca, Mexico Copenhagen IGWA, 1973 (Document 15)

BARTOLOME Leopoldo José O estrangeiro profissıonal e a tentação faustica a antropologia frente aos programas de desenvolvimento In ARANTES A A RUBEN G, DEBERT G (Org) Antropologia $e$ direttos humanos a responsabilidade do antropologo Campınas Ed da Unicamp, 1992 p 163174

BARTOLOME Leopoldo Jose BARANGER Dionisio Microproyectos de desarrollo rural en el nordeste argentino algunas lecciones In ENCUENTRO DE CIENTIFICOS SOCIALES, 3 UNAM UNIJOI UNOESC Posadas 1996 Anales Posadas Universidad Nacional de Misiones, Unıversidad del Oeste de Santa Catarina Unıversıdad do Noroeste de Rio Grande do Sul 1996 p 85100

CATULLO Marıa Rosa Podery partzcipacion en proyectos degran escala analisıs comparativos de los procesos de relocalizacion por la construccion 
de la represa binacional argentıno uruguaya de Salto Grande Tese (Doutorado em Ciênclas Sociaıs) - Instıtuto de Ciências Humanas, Unıversıdade de Brasilıa Brasilua, 1996

CERNEA Michael $M$ Why economic analysis is essential to resettlement a sociologist $\mathrm{s}$ view In____ (Ed) The economics of involuntary resettlement questions and challenges Washington DC (The World Bank Serıes on Directions and Development I999)

DE WET Chris C Can everybody win? economic development and population resettlement In CONGRESSO DA ASSOCIAÇÃO BRASILEIRA DE ANTROPOLOGIA RURAL 2000, Ofıcına sobre reassentamento involuntarıo riscos, reconstrução e desenvolvimento Rio de Janeiro Anals Rio de Janeiro 2000

FERNANDES, Walter, PARANJPYE, Vijay Rehabilitation policy and law in India a right to livelıhood New Delhı Indian Social Institute, 1997 GIDDENS, Anthony The constitution of soctety Berkeley University of Calıfornia Press, 1984

GOULD Stephens Jay Deconstructing the Science Wars by reconstructing an old mold Science, v 287 n 545 l p 2532612000 INTERNATIONAL COMMISSION ON LARGE DAMS (ICOLD) Ministerial Declaration of The Hague on water securty in the 21st Century 2000 Disponivel em < http //wwwworldwatercouncl org/fileadmin/wwc/ Library/Official_Declarations html > Acesso em 17 ago 2008

MANIBELI DECLA-ARATION Calling for a moratorium on World Bank funding of large dams Berkley CA International Rivers 1994 Disponivel em $<$ http //www internationalruvers org/en/follow money/manıbelideclaration html > Acesso em 17 ago 2008

PARTRIDGE, Willıam L , BROWN, Antoinnette Desarrollo agricola entre los Mazatecos reacomodados America Indigena, Mexico, v 43, n 2 p 3433621983

PARTRIDGE Willam L, BROWN, Antomnette Etnodesarrollo en la Mazateca America Indigena, Mexico, v 44 n 2 p 4054081984

SCUDDER Thayer Development induced improverishment, resistance and river basin development In MCDOWELL Christopher (Ed) Understanding improvershment the consequences of development induced displacement Providence Berghahn Books 1996 v 2, p 49-74

SCUDDER, Thayer Dam induced community resettlement prunciples and issues Capetown 1999 Memorandum prepared for the World Commis sion of Dams

WORLD BANK, THE Resettlement and development the bankwide review of projects involving involuntary resettlement (1986-1993) Washington, DC The Environment Department, 1994 